\title{
MEMBrane STRUCTURES AND THEIR USE IN CIVIL ENGINEERING
}

\author{
Nela FREIHERROVA ${ }^{1}$, Martin KREJSA ${ }^{2}$ \\ ${ }^{1}$ Department of Structural Mechanics, Faculty of Civil Engineering, VSB- Technical University of Ostrava, \\ Ludvika Podeste 1875/17, 70833 Ostrava - Poruba, Czech Republic \\ ${ }^{2}$ Department of Structural Mechanics, Faculty of Civil Engineering, VSB- Technical University of Ostrava, \\ Ludvika Podeste 1875/17, 70833 Ostrava - Poruba, Czech Republic \\ nela.freiherrova@vsb.cz, martin.krejsa@vsb.cz
}

DOI: $10.31490 /$ tces-2018-0008

\begin{abstract}
This paper is focused on the static analysis of membrane structures, which are typical for their ability to transfer only tensile forces. Membrane structures are becoming more popular owing to their lower initial cost and potential for use in structures with higher aesthetic claims. However, the designs of membrane face some challenges related to the specific properties of these support systems. This paper aims to analyse the current possibilities for using membrane structures and to evaluate these structures in terms of materials and design details and using numerical methods.
\end{abstract}

\section{Keywords}

Membrane, tension structure, fabric construction, geometric nonlinearity, numerical modelling, formfinding.

\section{Introduction}

Membrane structures are often designed as roofing for temporary buildings as well as permanent buildings. The use of membranes allows a very low weight, high shape variability, aesthetic airiness, and large spans. Another advantage is their low production and assembly requirements.

The design of such structures requires considerable experience and knowledge, as many sub-tasks need to be considered. Additionally, standards for membrane design have not yet been established [12]. It is necessary to design the initial shape, place the supporting ropes appropriately, and design the supporting structure.The design cannot be based on the Theory of small deformation because the equilibrium equations are applied to the undeformed structure. As the superposition principle is not valid, the calculation must be based on the Theory of large deformation.
Previously, membrane structures were dealt with through the minimal surface principle [11]. From the view of mechanics, the minimum surface is determined by an isotropic stress field that can be simulated as an analogue of soap bubbles. For given boundary conditions, a soap bubble captures shape with the minimum area between all the different surfaces.

When numerical methods for determining the initial shape were developed, physical modeling gradually declined. Today, there are special computational programmes for modelling. Some of these are based on single-step methods such as Grid method or Force density method, while some methods require iterative calculations, such as the Surface stress density method, Updated reference strategy (URS), Dynamic relaxation method. These methods allow for the design of more complex constructions than can be developed using physical modelling.

The aim of this study is to analyse the current possibilities for the use of membrane structures and to provide a summary of information concerning the materials, design details, and calculation methods for membrane structures. The development and history of membrane structures are discussed in Chapter 2. Two current, which have interesting sizes and shapes, are also presented. Options for the materials used in these structures are discussed Chapter 3. Chapter 4 describes important structural details and joints. A static analysis of membrane structures is given in Chapter 5, which also discusses the principles of the numerical methods used for design.

\section{Development of membrane structures}

Textile membrane structures have been used for thousands of years. They have served as homes for 
nomadic populations and shelters for soldiers. Later, membrane structures began to be used as roofs for pressurised halls. However, Vladimir Grigorjevic Schuchov (28. 8. 1853 -2.2. 1939) can be considered the first pioneer of membrane structures. He was a Russian scientist, engineer, and architect. His scientific works in the early 20th century were systematic studies of uneven surfaces, rod structures, and membranes. At the AllRussian Exhibition in Nizhny Novgorod (1896), he presented eight pavilions with very large spans. Most notable was the construction of the Shuchov rotunda (see Fig. 1). The roofing of the Shuchov rotunda was constructed of a dense rope structure covered with a thin membrane. [2]

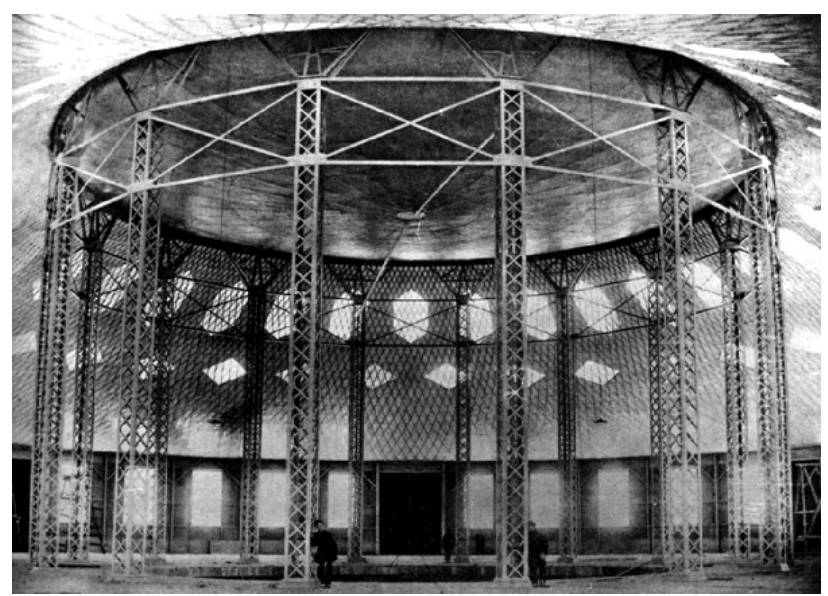

Fig. 1: Shuchov rotunda, Andrei Karelin 1896

Of current buildings, it is worth mentioning the dome with the largest cupola in the world, the Millennium Dome (see Fig. 2), which was opened on the occasion of the beginning of the new millennium. The span of the Millennium Dome is a symbolic $365 \mathrm{~m}$ (the same number as the number of days in a year). The roof is suspended on twelve supports which are $95 \mathrm{~m}$ high and the dome reaches a height of $50 \mathrm{~m}$. A teflon coated glass fibre fabric was used for the membrane. [1]

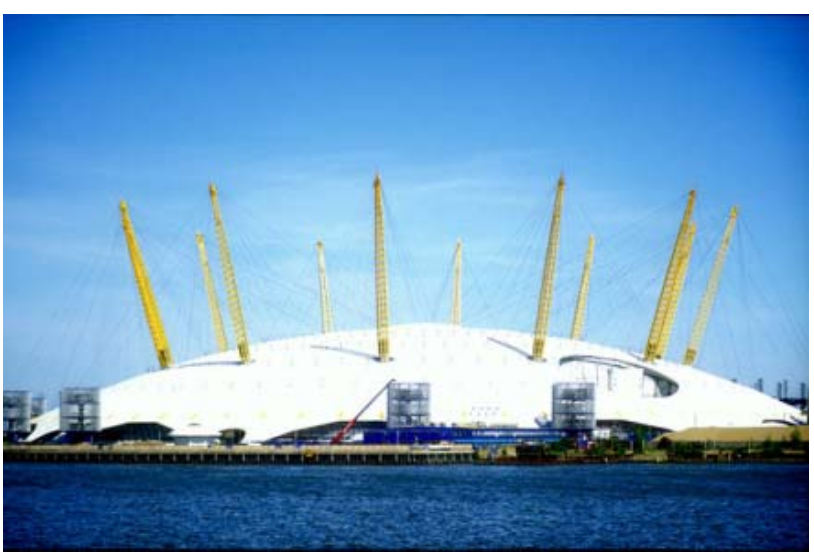

Fig. 2: Millennium dome, London[1]

In the Czech Republic, there is an interesting membrane construction at the Bruntal bus station, which was completed in 2015 (see Fig. 3). This building is interesting because its shape is in the form of 100-ton wings, and it also has unusual dimensions. The height of the roof structure is $4.75 \mathrm{~m}$ at the lowest point. At its highest point, the roof reaches $7.35 \mathrm{~m}$, and the clear height of the platform is $3.37 \mathrm{~m}$. During the design process, three static assessments were performed to demonstrate the safety of the roofing. The project was entered into a competition called the "Czech Republic and Slovakia Tekla BIM Awards 2014" and received a special award for its steel construction. The author of the original roofing is Ing. arch. Denisa Barvikova.

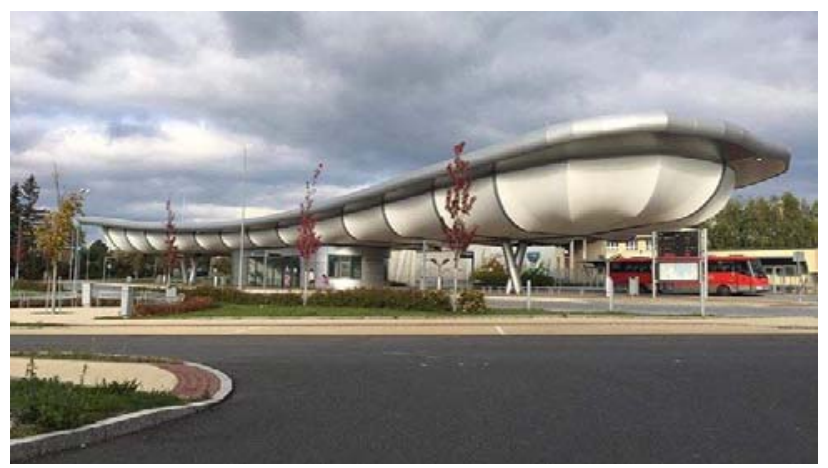

Fig. 3: Bus station in Bruntal, 2018

\section{Materials}

\subsection{Textiles}

The monolayer membrane materials in use today are typically made of polyester, PTFE, or glass or aramid fibre materials. Polyesters (or other fibres) provide stiffness and strength to the membrane. They are complemented with a PVC, PTFE, or silicone coating to protect against UV radiation and other atmospheric influences.

The membrane fabric transmits the load through its normal stiffness (its bending stiffness is negligible), which is variable depending on the stress state. Biaxial tests are used for evaluating, testing, and verifying the properties of materials. During these tests, stresses are measured in two directions using strain gauges and stretching. For the measurements, track sensors with a range corresponding to the expected transformation are used. The result of these tests is a plot in which differences in material behaviour can be observed in two main directions (warp vs. weft). The modulus of elasticity can also be derived using another calculations. Hovewer, it is variable due to the stress state. For polyester-based materials, values of the modulus of elasticity are about $1000 \mathrm{MPa}$

The thickness of the textile materials used can vary from $0.5-1.5 \mathrm{~mm}$, their weight can range from $0.5-2$ $\mathrm{kg} / \mathrm{m}^{2}$, and their tensile strength can vary from 300-1200 $\mathrm{daN} / 5 \mathrm{~cm}$ (i.e. the tensile strength in dekanewtons of a strip with a width of $5 \mathrm{~cm}$ ) [1]. 


\subsection{Ropes}

Wire ropes fabricated from single wires $(1 \times 19,1 \times 37$, or $1 \times 61)$ are commonly used for membrane structures. The material strength of the wire is approximately $1500 \mathrm{MPa}$. However, the material strength of the rope is smaller than that of the wire, and the reduction in parameters also affects the calculation of reliability. The modulus of elasticity of the ropes can reach $160,000 \mathrm{MPa}$. [1]

Nowadays, synthetic ropes can also be used. Compared to steel ropes, synthetic ropes have better fatigue and damping properties, and have a high strengthto-weight ratio. On the other hand, disadvantages of synthetic ropes include a lower modulus of flexibility and high sensitivity to rheological influences.

\section{Construction details and joints}

Generally, supports for membrane structures can be divided into two basic types: line (see Fig. 4) and point (see Fig. 5).

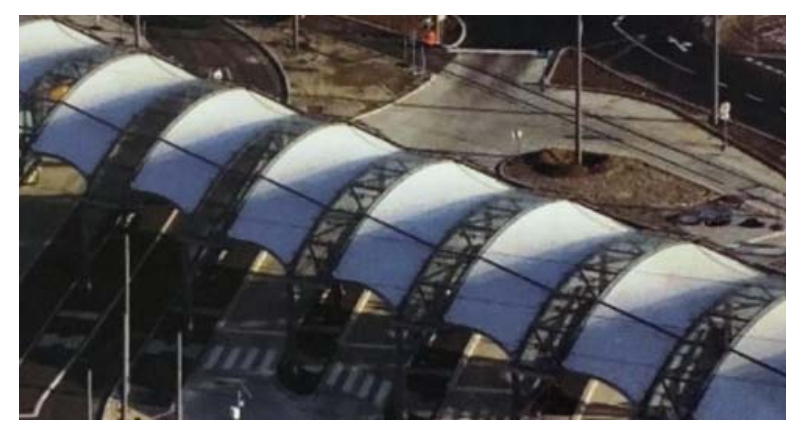

Fig. 4: Line-type supports[1]

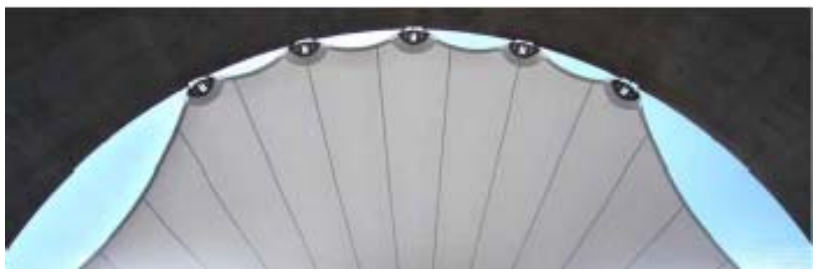

Fig. 5: Point-type supports [1]

Line anchorages are constructed using Keder rails that slide into an auxiliary profile. They are then fixed to the supporting structure with conventional fasteners (see Fig. 6).

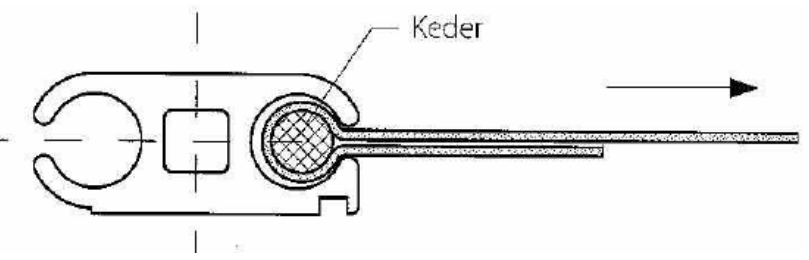

Fig. 6: Keder rails in an auxiliary profile for line anchoring [1]
In the case of point anchoring, Keder rails are also used. They are clamped between two steel plates of the corner joint of the fabric (see Fig. 7).

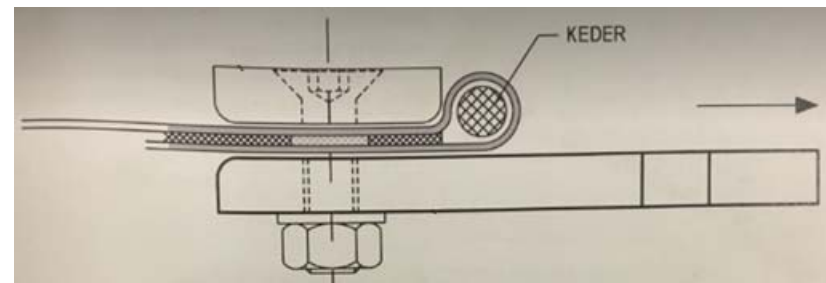

Fig. 7: Keder rails in an auxiliary profile for point anchoring [1]

For corner anchor assemblies, it is very important to attach the membrane's corner sheet to the column joint or subsequent structure, correctly. The so-called "checkers" are used and they allow rotation around one axis. A double check is used, if it is necessary to allow rotation around the second axis in the storage. In this case, this element forms an omni-directional joint (see Fig. 8).

Another possible corner anchorage uses a fork with a die-cutter terminal. Attachment to the corner profile is achieved through the pipe which is part of the terminal (see Fig. 9).

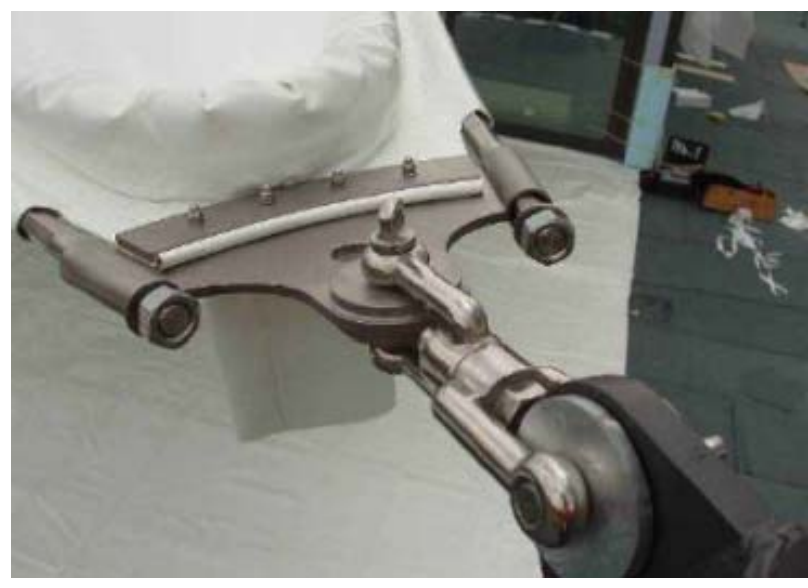

Fig. 8: Connection between the corner plate of a membrane and the joint plate [1]

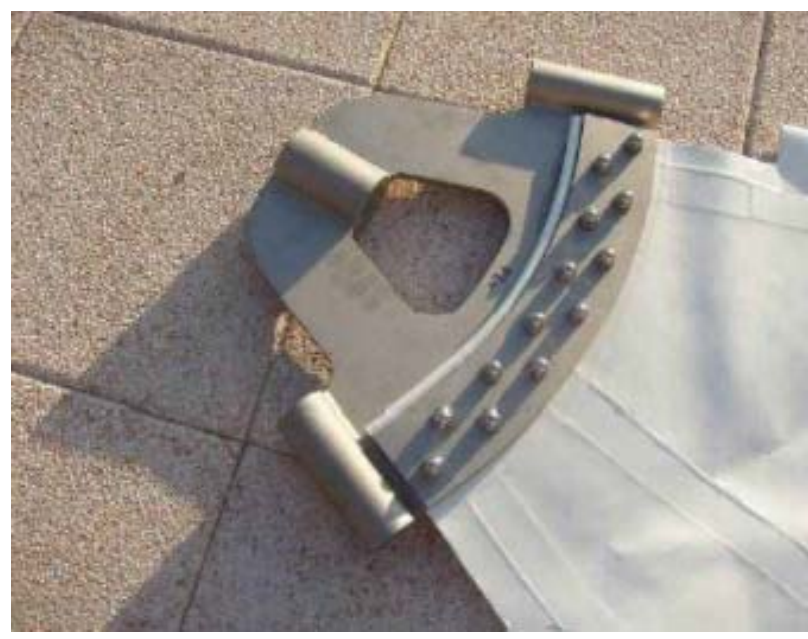

Fig. 9: Corner anchoring using a fork with a die-cutter terminal [1] 
Point details for fastening peripheral ropes are also part of the corner point anchorage. Ropes can be held in place in pockets (see Fig. 10) for smaller forces in the diaphragm or with a clamping system for cases in which larger forces need to be transferred (see Fig. 11).

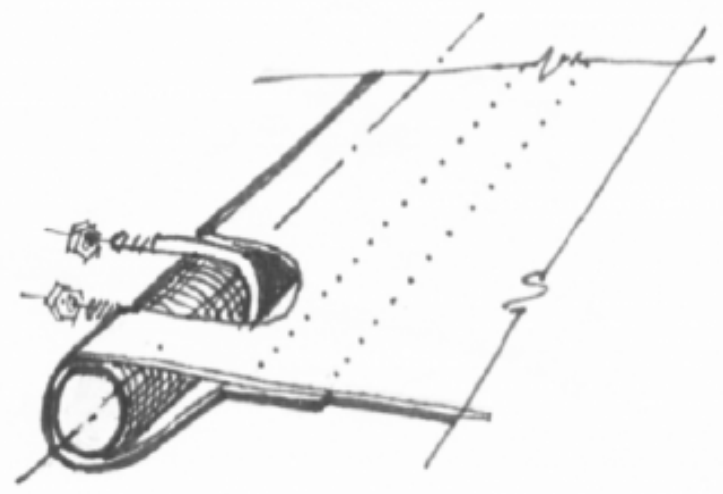

Fig. 10: Peripheral ropes held in place with pockets [21]

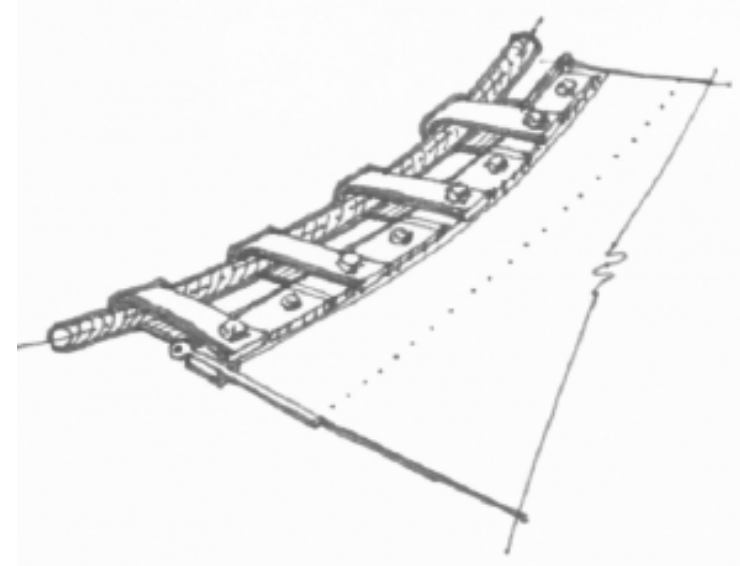

Fig. 11: Peripheral ropes held in placewith a clamping system [21]

The ropes are tightened with the threaded terminal and nuts through the pipes of the corner profile. The optimal pre-stress and shape of the fabric can be achieved without unwanted wrinkling.

For the top part of the membrane, steel rings can be used. These also need to satisfy the requirements for waterproof construction.

In general, the bonding of the membrane material can be divided into two types: permanent joints (welded, combined, or glued) and temporary or reusable joints (clamping plates and Keder rails).

The most common of these are welded joints. They can be overlapped with different overhangs or employed as contact joints with a cover strip. Welded joints are usually made in a workshop, and their strength is approximately $60-95 \%$ the strength of the fabric, depending on the welding process and temperature. These joints are waterproof and UV stable.

Reusable joints are used for the assembly joints of the membrane fabric owing to its maximum production and transport dimensions; they are also used for mounting the membrane to the construction. In particular, these types of joints are carried through steel bushings.

More detailed informations about materials and construction elements can be found in [7].

\section{Static analysis of membrane structures}

When analysing membrane structures, their geometric non-linearity must be taken into account [4]. The resulting deflections are many times greater than the thickness of the materials, and the necessary calculations cannot be performed based on The Theory of small deformation. The principle of superposition does not apply, and it is necessary to load the structure over the whole range and not as individual load cases.

By 1969, physical models (using soap bubbles, fabrics, or ropes) were being used for the design of membrane structures. A major milestone in this era was the roof of the stadium for the 1972 Summer Olympics in Munich. German architect Otto Frei was one of the designers. His publication [3], supplemented by a number of physical contexts, was crucial foradvances in numericalmodelling.

Facilitated by advances in computer technology, numerical modelling has been performed using special numerical software. These models can determine the initial equilibrium state of the membrane based on selected boundary conditions, materials, prestress levels, and loads.

Currently available methods for determining the equilibrium allow the design of structures with larger dimensions and more interesting shapes.

\subsection{Basic characteristics of membrane structures}

Membrane structures are able to transmit loads only after a prestress has been applied and a suitable shape achieved. These structures can be divided into the following categories:

- $\quad$ single-skinned,

- two or more coats,

- directly prestressed (e.g. by inserting membrane forces),

- $\quad$ indirectly prestressed (ie overpressure),

- freely prestressed, stabilised by rigid construction,

- $\quad$ freely prestressed, stabilised by ropes,

- prestressed and stabilised by air pressure, underpressure or overpressure, 
- $\quad$ prestressed with light gas and stabilised by a rope system,

- hybrid.

The spatial effect of prestressing is determined by the curvature, and is performed in two oppositely oriented planes. Membrane structures can be divided into the following basic shapes:

- $\quad$ hyperbolic paraboloid (see Fig. 12),

- $\quad$ cone (see Fig. 13),

- $\quad$ saddle (see Fig. 14),

- $\quad$ overpressure membranes.

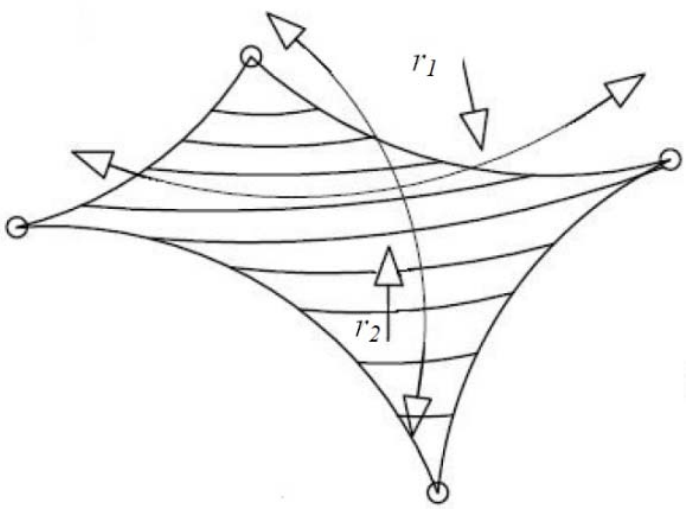

Fig. 12: Hyperbolic paraboloid shape

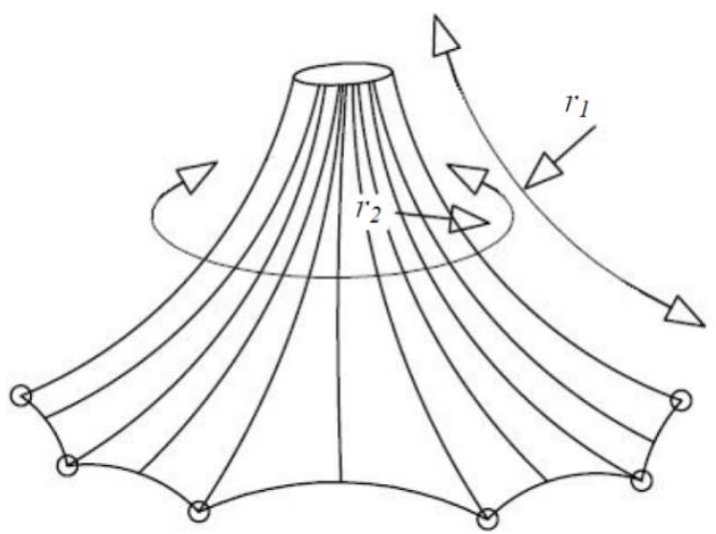

Fig. 13: Cones hape

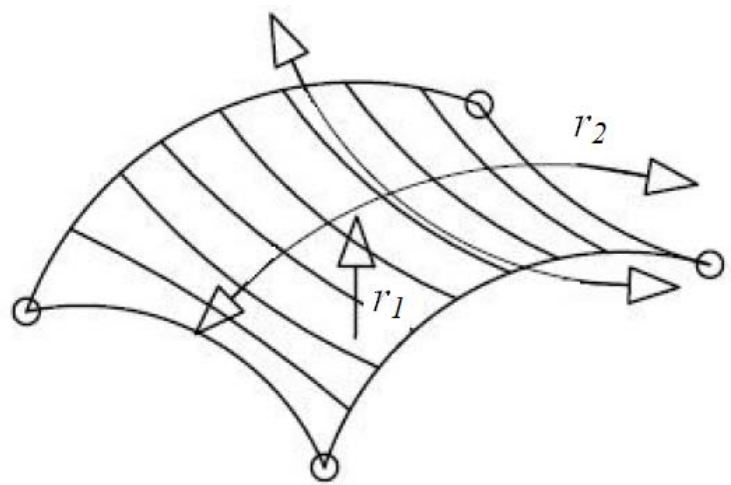

Membrane structures can also be divided according to the used material and the duration of the construction.

\subsection{Prestressing}

The stiffness and stability of membrane structures are determined by the radius of curvature in two directions and by the prestressing. Prestressing prevents inversion of the curvature after the load is applied to the structure. Thus, prestressing is the basic loading state, and it activates the structure's anchor structure, stiffness, and ability to carry the load. The prestressing value is typically in the range of $0.5-2 \mathrm{kN} / \mathrm{m}^{2}$. However, for larger spans, the prestressing value can reach $5 \mathrm{kN} / \mathrm{m}^{2}$ [1].

For the construction, the appropriate size is determined through a process of searching for the optimal shape, called Form-finding [16]. The minimum required prestress of the membrane is based on the required stiffness and strength of the material. The prestress value should also consider long-term effects such as creep of the membrane and settling of the foundation structure.

Prestressed ropes are used to determine additional boundary conditions. The higher the forces in the ropes, the smaller their curvature will be. On the other hand, the surface of the membrane will increase (see Fig. 15).

The straighter the rope is, the greater the force transferred to the supporting structure and anchoring will be.
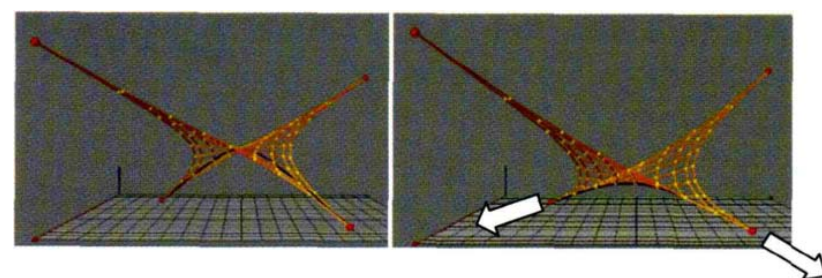

Fig. 15: Effect of the magnitude of the force in the ropes on the resulting membrane surface [15]

Another parameter which affects the resulting stress and curvature is the placement of the prestressed ropes (see Fig. 16). The location of the ropes affects the equilibrium of the construction.

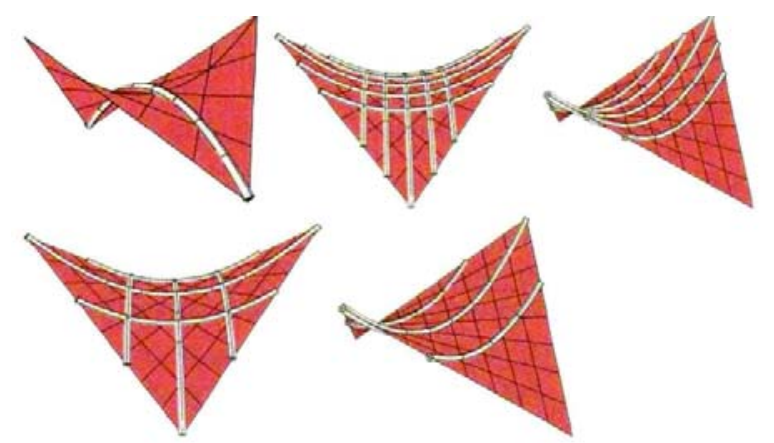

Fig. 16: Possible placements of the prestressed ropes in a structure [15]

Fig. 14: Saddle shape 


\subsection{Numerical methods for membrane structures}

\section{1) Grid method}

This method is based on the assumption of a balance of horizontal forces. The $z$-coordinates of the network nodes are obtained by determining the balance of vertical forces. Membrane structures are modelled using a quadrangle rope network. The Grid method assumes that the perpendicular projections of the ropes are orthogonal at each node, and the node connectors are lines.

Figure 17 shows the realisation of the mesh projection in the $x-y$ plane during modeling of a membrane.

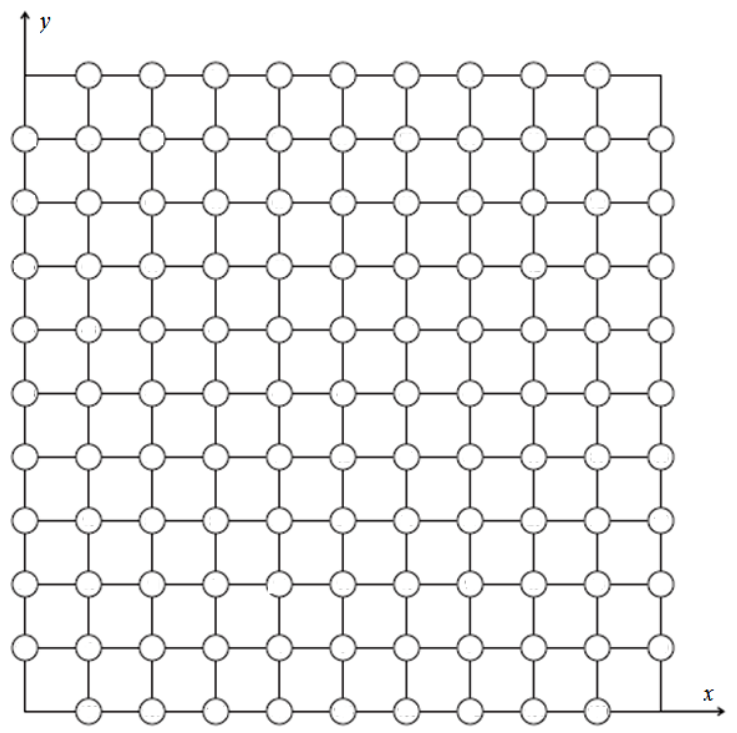

Fig. 17: Network layout using the Grid method

Then, for each node (see Fig. 18), the three nonlinear equilibrium equations are determined (see Eqs. 1, 2, and 3 ), in which $x_{i}, y_{i}$, and $z_{i}$ are the coordinates of node $i$ (corresponding variables refer to the other nodes: $j, k, l$, and $m), F$ is the vector of the external force acting on a particular node in the given direction, $L_{i j}$ is the length of the connection between nodes $I$ and $j$, and $T_{i j}$ is the magnitude of the stress vector of node acting on node $i$. Because an assumption of zero horizontal forces is made, $F_{x i}$ and $F_{y i}=0$.

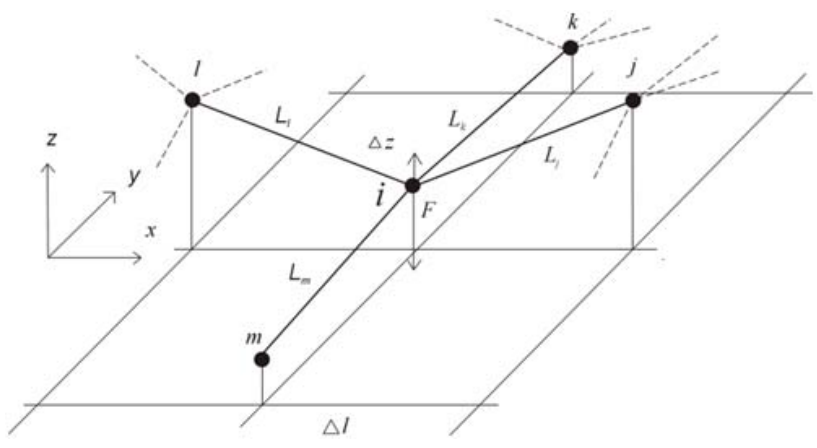

Fig. 18: Node diagram created using the Grid method
$T_{i j} \frac{x_{j}-x_{i}}{L_{i j}}+T_{i k} \frac{x_{k}-x_{i}}{L_{i k}}+T_{i l} \frac{x_{l}-x_{i}}{L_{i l}}+T_{i m} \frac{x_{m}-x_{i}}{L_{i m}}+$

$+F_{x i}=0$,

$T_{i j} \frac{y_{j}-y_{i}}{L_{i j}}+T_{i k} \frac{y_{k}-y_{i}}{L_{i k}}+T_{i l} \frac{y_{l}-y_{i}}{L_{i l}}+T_{i m} \frac{y_{m}-y_{i}}{L_{i m}}+$

$+F_{y i}=0$,

$$
\begin{aligned}
& \quad T_{i j} \frac{z_{j}-z_{i}}{L_{i j}}+T_{i k} \frac{z_{k}-z_{i}}{L_{i k}}+T_{i l} \frac{z_{l}-z_{i}}{L_{i l}}+T_{i m} \frac{z_{m}-z_{i}}{L_{i m}}+ \\
& +F_{z i}=0 \text {. }
\end{aligned}
$$

The equilibrium state of the horizontal orthogonal forces can be derived from the three nonlinear equations (see Eqs. 4 and 5), where $\Delta l$ is the pitch of the grid.

$T_{i j} \frac{\Delta l}{L_{i j}}-T_{i l} \frac{\Delta l}{L_{i l}}=0$,

$T_{i k} \frac{\Delta l}{L_{i k}}-T_{i m} \frac{\Delta l}{L_{i m}}=0$.

Therefore, the magnitude of the horizontal components in $x$-and $y$-directions is constant, owing to the following:

$\frac{T_{i j}}{L_{i j}}\left(z_{j}-2 z_{i}+z_{l}\right)+\frac{T_{i k}}{L_{i k}}\left(z_{k}-2 z_{i}+z_{m}\right)+F_{z i}=0$.

This equation can be derived for each node. The result is a set of linear equations for unknown coordinates in the $z$-direction.

The calculated area of a membrane for a network with unchanged boundary coordinates is shown in Fig. 19.

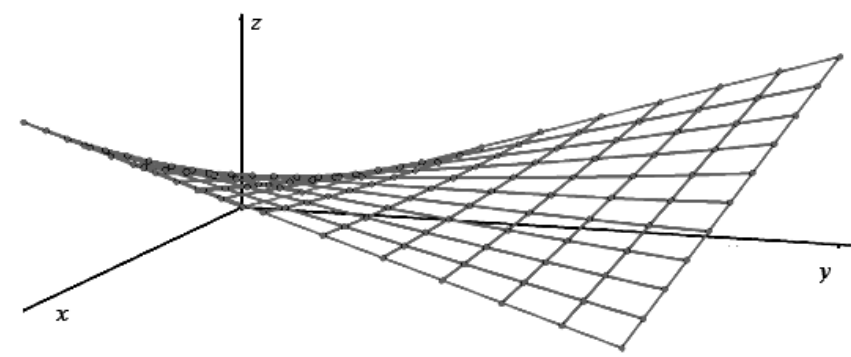

Fig. 19: Net result obtained using the Grid method with suitably chosen input conditions

More detailed information about this method can be found in [8].

\section{2) Force density method}

The Force density method can be considered a generalised Grid method. Again, a network consisting of nodes and connectors (ropes or rods) is established. If the network is hung on a fixed frame or between fixed points, it remains in the equilibrium position, and the sum of the forces at each node is zero.

Unlike the Grid method, the orthogonal mesh does not have to be perpendicular, and the calculation affects all three nodal coordinates. Determination of the mesh can also be performed topologically (see Fig. 20). 

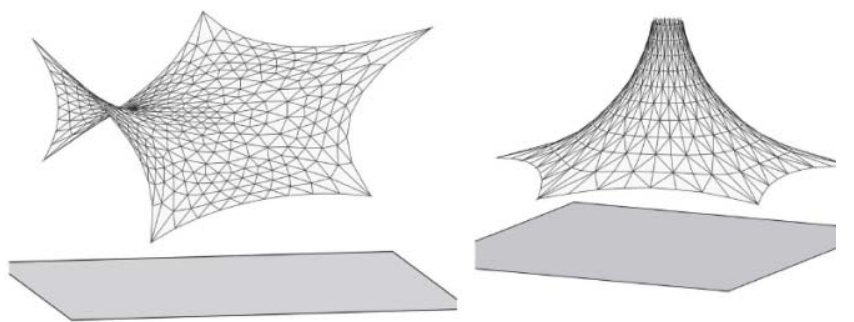

Fig. 20: Examples of a topological network for using the Force density method [17]

For finding the coordinates of node $i$, which is connected by lines to nodes $j, k, l$, and $m$, it is necessary to consider the dependence on the lengths $L_{i j}, L_{i k}$, and $L_{i l}$. A new magnitude, called the force density, which indicates the ratio between the force $T_{i j}$ and length $L_{i j}$, is introduced into the calculation (see Eq. 7).

$q_{i j}=\frac{\mathrm{T}_{\mathrm{ij}}}{\mathrm{L}_{\mathrm{ij}}}$

The equilibrium equations then have the following form:

$q_{i j}\left(x_{j}-x_{i}\right)+q_{i k}\left(x_{k}-x_{i}\right)+q_{i l}\left(x_{l}-x_{i}\right)+$

$+q_{i m}\left(x_{m}-x_{i}\right)+F_{x i}=0$,

$q_{i j}\left(y_{j}-y_{i}\right)+q_{i k}\left(y_{k}-y_{i}\right)+q_{i l}\left(y_{l}-y_{i}\right)+$

$+q_{i m}\left(y_{m}-y_{i}\right)+F_{y i}=0$,

$q_{i j}\left(z_{j}-z_{i}\right)+q_{i k}\left(z_{k}-z_{i}\right)+q_{i l}\left(z_{l}-z_{i}\right)+$

$+q_{i m}\left(z_{m}-z_{i}\right)+F_{z i}=0$,

where $q_{i j}, q_{i k}, q_{i l}$, and $q_{i m}$ represent the force densities. Because $q_{i}$ is constant, the calculation leads to a set of three linear equations for the $x, y$ and $z$ coordinates, which are then obtained with the matrix number.

The disadvantage of the Force density method is that it the lengths of the ropes, which are necessary for the calculation, are not always known. This method is also used by the surface stress density method discussed in the following section.

More detailed information about the force density method can be found in [9], [13], [14].

\section{3) Surface stress density method}

This method neglects the shear stresses and models the membrane using triangular elements (see Fig. 21).

The difference between this method and the methods above is that the Surface stress density method provides information about the stress distribution in the membrane and not just for the ropes of the mesh. The material of the membrane is as assumed to be isotropic (i.e. having the same properties in all directions). Because the shear stress is neglected and the membranes are two-dimensional problems, the stress tensor can be written as follows:

$$
\begin{aligned}
\sigma & =\left(\begin{array}{lll}
\sigma_{11} & \sigma_{12} & \sigma_{13} \\
\sigma_{21} & \sigma_{22} & \sigma_{23} \\
\sigma_{31} & \sigma_{32} & \sigma_{33}
\end{array}\right)=\left(\begin{array}{ccc}
\sigma_{11} & \sigma_{12} & 0 \\
\sigma_{21} & \sigma_{22} & 0 \\
0 & 0 & 0
\end{array}\right)= \\
& =\left(\begin{array}{ccc}
\sigma_{0} & 0 & 0 \\
0 & \sigma_{0} & 0 \\
0 & 0 & 0
\end{array}\right)=\left(\sigma_{0}\right) .
\end{aligned}
$$

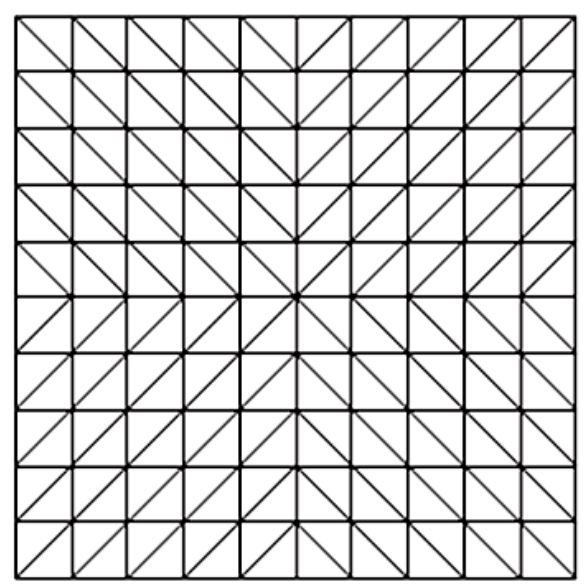

Fig. 21: Triangular mesh used for the Surface stress density method

The following is a given:

$T_{i}=L_{i} H \sigma_{0}$,

where $H$ is the thickness of the membrane, $T_{i}$ is the magnitude of the forces at the corners of the triangular mesh element perpendicular to the opposite side with length $L_{i}$ (see Fig. 22).

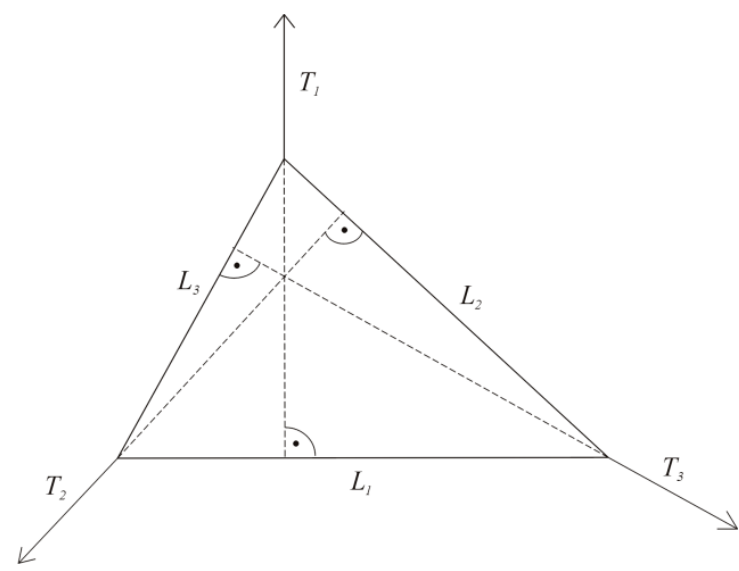

Fig. 22: Forces at the vertices of a triangular element

For each node, its stress vector can be expressed, which is the effect of the triangular membranes on each of their vertices.

For $n$ triangular elements adjacent to node, it is necessary to express the total internal force expressed in the global system (see Fig.23). This force is the sum of the forces from all of the triangular elements of the mesh acting on this point (see Eq. 13). This force can be decomposed with respect to the coordinate axes (i.e. into $F_{c x}, F_{c y}$, and $F_{c z}$ ), and the partial expressions can be used 
to obtain the coordinates of point $\mathrm{C}\left(x_{c}, y_{c}\right.$, and $\left.z_{c}\right)$.

$F_{C}=\sum_{i=1}^{n} T_{C i}=\sum_{i=1}^{n} Q_{S i} L_{i}^{2} \overrightarrow{C P_{l}}$

where $Q_{S i}=\frac{\sigma_{0_{i}}}{S_{i}}$.

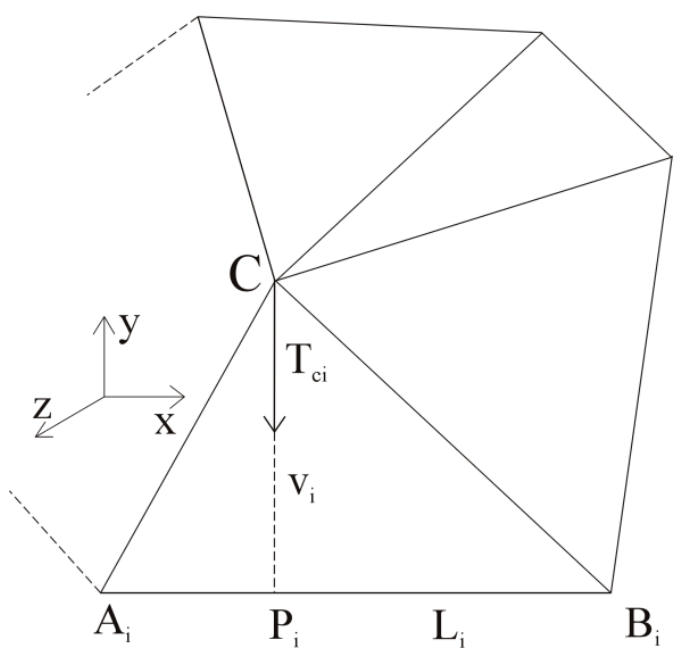

Fig. 23: Internal nodal forces acting on point $C$

To achieve static equilibrium, the condition $F_{c}=0$ must be satisfied. The calculation is an iterative process which continues until this condition is met.

More detailed information about this method can be found in [10].

\section{4) Updated reference strategy (URS)}

The equilibrium state of the membrane is defined by the principle of virtual work. It is assumed that the sum of the virtual work performed by the required prestress, $\sigma$, and the external load, $p$ (e.g. overpressure, the weight of the structure itself), must be zero.

In solving the problems of the principle of virtual work, it has been shown that the matrix of the solved unknown parameters is singular due to the inverse nature of the problem.

The URS method solves the singularity problem by introducing an expanding variable which takes into account the formulation of the principle of virtual work.

In creation of the equilibrium state, it is necessary to find the configuration in which the surface Cauchy stress, $\sigma$ (i.e. the actual stress measured at a given moment on the deformed body) is replaced by the second PiolaKirchhof stress, $S$, which is related to the undeformed body. Because these two stresses are artificially connected by a tangential mesh deformation, there is no singularity when using $S$. If there is no deformation, $\sigma$ and $S$ are identical.

Consider a field of tension that acts in the plane tangential to the surface in equilibrium (see Fig.24). The stress field is thus the total stress resulting from the initial prestress and the deformation of the textile membrane without fixed boundaries.

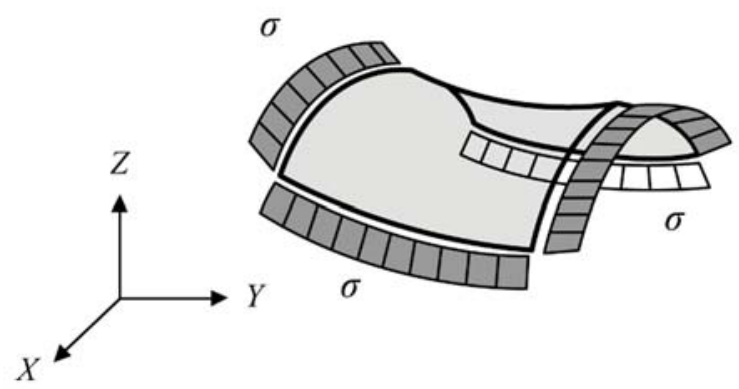

Fig. 24: Stress in the tangential plane

At this point, it is not necessary to specify a particular material because the stress field is considered a given.

It is necessary to determine the geometry of the surface that allows this tension to be in equilibrium. When using this method, the equilibrium state of the membrane is defined by the principle of virtual work as follows:

$\delta w=t \int_{a} \sigma: \frac{\partial(\delta u)}{\partial x} d a=t \int_{a} \sigma: \delta u_{x} d a=0$,

where $a$ is the minimal surface, $\delta$ is the Cauchy stress tensor, which is applied to the surface in equilibrium; $\delta_{u, x}$ is the derivative of the virtual displacement with respect to the geometry of the current surface; and $t$ is the thickness of the membrane, which is relatively thin and assumed to be constant during deformation; the Poisson's coefficient of the thickness is neglected. Equation 15 expresses that the virtual work of the given stress field is zeroat equilibrium.

To express $\delta_{u x}$, the deformation gradient, $F$, is introduced into the calculation. The deformation gradient includes the volume and shape changes of the deformed body. It defines the actual shape of area $a$ by the deformed shape of a reference configuration of area $A$. It satisfies the following expression:

$\delta u_{x}=\frac{\partial(\delta u)}{\partial x}=\frac{\partial(\delta x)}{\partial X} \cdot \frac{\partial X}{\partial x}=\delta\left(\frac{\partial x}{\partial X}\right) \cdot F^{-1}=\delta F \cdot F^{-1}$

After substituting the equation for the deformation gradient into the equation for the expression of virtual work with subsequent modifications, the following expressions can be obtained:

$\delta w=t \int_{a} \sigma: \delta u_{x} d a=t \int_{A} \sigma: \delta u_{x} \operatorname{det} F d A=$

$=t \int_{A} \sigma:\left(\delta F \cdot F^{-1}\right) \operatorname{det} F d A=0$

$\rightarrow$

$\delta w=t \int_{A} \sigma:\left(\delta F \cdot F^{-1}\right) \operatorname{det} F d A=$

$=t \int_{A} \operatorname{det} F\left(\sigma \cdot F^{-T}\right): \delta F d A=0$

$\rightarrow$

$\delta w=t \int_{A} \operatorname{det} F\left(F \cdot F^{-1} \cdot \sigma \cdot F^{-T}\right): \delta F d A=$

$=t \int_{A}\left(F \cdot\left(\operatorname{det} F \cdot F^{-1} \cdot \sigma \cdot F^{-T}\right)\right): \delta F d A=$

$=t \int_{A}(F . S): \delta F d A=0$,

where $S$ is the second Piola-Kirchhoff stress, which replaced the surface Cauchy stress, $\sigma$. However, the $S$ elements are related to the original configuration and the 
$\sigma$ elements are related to the current configuration. If the current and original configurations are the same, then $S$ and $\sigma$ will be identical [11], [17].

\section{5) Dynamic Relaxation Method (DRM)}

DRM is an iterative process that is used for the static analysis of structures. This method is not used for the dynamic analysis of structures; a dynamic solution is used for a fictitious damped structure to achieve a static solution.

The method relies on a discretized continuum. The mass of the structure is assumed to be concentrated at given points (nodes) of the structure in this a discretized continuum. At these nodes, residual forces (the difference between internal and external forces) are also calculated and nodal displacements are calculated on the basis of Newton's second law of motion, in which residual forces and fictitious variables are used.

The nodal displacement equation may be written for each node separately, because the diagonal mass matrix and the diagonal damping matrix attenuation are considered. DRM does not require the assembly and storage of the global stiffness matrix of the structure which is the main advantage of this method.

More detailed information about this method can be found in [5], [6].

\subsection{Software}

Nowadays, specialised programmes are used for the design and analysis of membrane structures.

A commonly used softwareis the Easy software created by Technet [19]. This software allows the design of mechanically prestressed membranes and overpressure membranes, including the supporting structures.

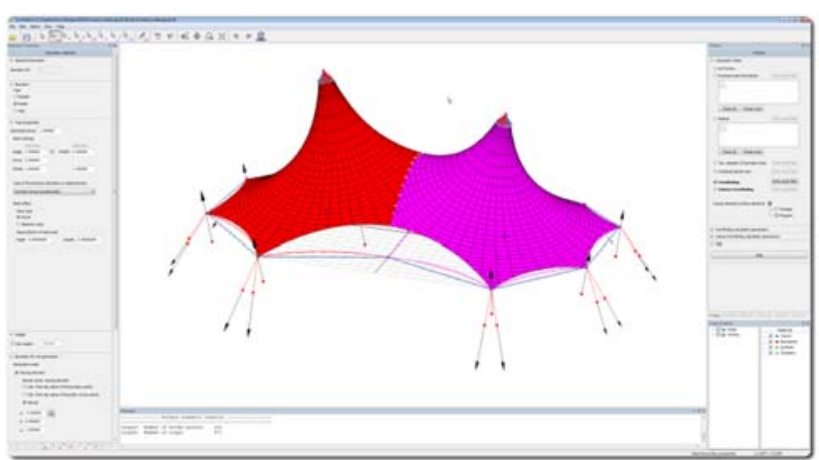

Fig. 25: The Easy softwarefor the design of membrane structures[20]

Another available programme is the RFEM software created by the Czech company, Dlubal [20]. An additional $\mathrm{RF}$ form-finding module was created to calculate membrane structures. It allows determination of the initial shapes of membrane and rope structures, which are given by physical and geometric boundary conditions and equilibrium prestress; eventually, the equilibrium system can be determined with the prestress and load. In the form-finding process, the URS method is applied.

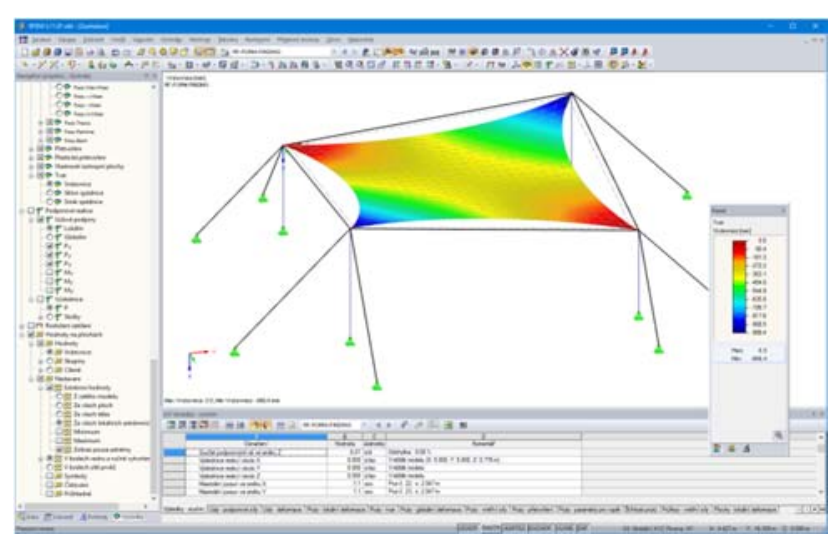

Fig. 26: Software system with RF form-finding module [21]

Other software options include Meliar Mpanel, Membrane NDN, and Forten 4000 [1].

\section{Conclusion}

The aim of this paper was to analyse the current possibilities for using membrane structures because their design is not simple and designers must overcome a number of pitfalls resulting from the specific properties of these supporting systems. This paper discusses the analysis of suitable materials and selected structural details. Possible calculation methods are also described, along with their theoretical background and applications in commercial programmes for membrane design.

Owing to the lack of a standard for the design of membrane structures, the designer must rely on a determination of the stress and strain by means of numerical modelling with verification through physical tests. Therefore, further research on membrane structures should focus on investigation of the stress-strain states of the most common types of membrane structures with the goal of optimising their shape and analysing the suitability of their use for building construction purposes.

\section{Acknowledgements}

This contribution has been completed thanks to the financial support provided to VSB-Technical University of Ostrava by the Czech Ministry of Education, Youth and Sports from the budget for conceptual development of science, research and innovations for the 2018 year.

\section{References}

[1] KOPRIVA, Milos, Michal NETUSIL, Henri ACHTEN and Zdenek HIRNSAL. Membrane Architecture. CTU in Prague, 2015. ISBN 978-8001-05693-6 (in Czech). 
[2] VENTSEL, Eduard and Theodor KRAUTHAMMER. Thin plates and shells: theory, analysis, and applications. New York: Marcel Dekker, 2001,666 p. ISBN 0824705750.

[3] FREI, Otto. Grundbegriffe und Übersicht der zugbeanspruchten Konstruktionen Band 2. Ullstein, 1962.

[4] GALISHNIKOVA, Vera, Peter DUNAISKI and Peter Jan PAHL. Geometrically Nonlinear Analysis of Plane Trusses and Frames. Stellenbosch: SUN MeDIA, 2009. ISBN 978-1-920109-48-6.

[5] HUTTNER, Milos, Jiri MACA and Petr FAJMAN. The efficiency of dynamic relaxation methods in static analysis of cable structures. Advances in Engineering Software. 2015(89), 28-35. DOI: 10.1016/j.advengsoft.2015.06.009.

[6] HUTTNER, Milos, Jiri MACA and Petr FAJMAN. Analysis of cable - membrane structures using the Dynamic Relaxation Method. IX International Conference on Structural Dynamics. Portugal, Book Series: EURODYN 2014, 2014, 1919-1926. ISSN 2311-9020.

[7] SEIDEL, Michael. Tensile Surface Structures. A Practical Guide to Cable and Membrane Construction: Materials, Design, Assembly and Erection. Berlin: Wiley VCH, 2009. ISBN 9783433029220 .

[8] SIEV, Avinadav and J. EIDELMAN. Stress Analysis of Prestressed Suspended Roofs. Journal of the Structural Division. 1964, 90(4), 103-122.

[9] SCHEK, Hans Jürg. The force density method for form finding and computation of general networks. Computer Methods in Applied Mechanics and Engineering. Elsevier B.V., 1974, 3(1), 115-134. DOI: $10.1016 / 0045-7825(74) 90045-0$.

[10] MAURIN, Bernard and Rene MOTRO. The surface stress density method as a form-finding tool for tensile membranes. Engineering Structures. France, 1998, 20(8), 712-719. DOI: $10.1016 / \mathrm{S} 0141$ 0296(97)00108-9.

[11] WÜCHNER, Roland and Kai-Uwe BLETZINGER. Stress-adapted numerical form finding of pre-stressed surfaces by the updated reference strategy. Numerical methods in Engineering. John Wiley, 2005, 64(2), 143-166. DOI: $10.1002 /$ nme. 1344 .

[12] PYLA, Lincy, Xiduo WANGB, Elien DE SMEDTC, Jimmy COLLIERS, Marijke MOLLAERT and Lars DE LAET. Existing Eurocodes Applied to a Membrane Structure. Procedia Engineering. Elsevier, 2016, 155, 142 - 151. DOI: 10.1016/j.proeng.2016.08.015.

[13] PINA, Rafael Jurado and M. A. Salazar TROYA. A simple method for the design of tension structures combining topological mapping and nonlinear structural analysis. Informes de la construcción. 2014, 66 (Extra 1).DOI: 10.3989/ic.13.090. ISSN 0020-0883.

[14] KOOHESTANI, Kambiz. Nonlinear force density method for the form-finding of minimal surface membrane structures. Communications in Nonlinear Science and Numerical Simulation. 2014, 19(6), 2071-2087. DOI: 10.1016/j.cnsns.2013.10.023.

[15] PRONK, Arno and D. MAURICE. 84 Ways to Manipulate a Membrane. Journal of the International Association for Shell and Spatial Structures. International Association for Shell and Spatial Structures (IASS), 2012, 53(12), 257-270. ISSN 1028365X.

[16] NEMEC, Ivan and ROSTISLAV Lang. Design and Analysis of the Membrane Structure. Transactions of the VSB - Technical University of Ostrava, Mechanical Series 1/2013, 2013.

[17] WRIGGERS, Peter and Paolo DE MATTOS PIMENTA: New Trends in Thin Structures: Formulation, Optimization and Coupled Problems. Springer Verlag, 2010.ISBN 978-3-7091-0231-2.

[18] LANG, Rostislav, NEMEC, Ivan and Ivan SEVCIK. Form- finding of membrane structures with regard to its specific necessities. In Proceedings of the 6th international conference Dynamics of Civil Engineering and Transport Structures and Wind Engineering, DYN-WIND 2014.2014. s. 162 169. ISBN: 978-80-554-0844- 6.

[19] Technet GmbH [online]. [cit. 2018-09-22]. Available from: https://www.technet-gmbh.com/

[20] Software for the calculation of statics of building structures Dlubal [online]. Dlubal Software, 2001 [cit. 2018-09-22]. Available from: https://www.dlubal.com/cs

[21] Fabric Architecture: Tension structure connection details [online]. USA: Industrial Fabrics Association International, 1970, 2010(January) [cit. 2018-10-12]. Dostupné http://www.aerosail.com/PDF/Tension_structure_co nnection details Fabric Architecture.pdf 